\title{
Renal Transplantation in Patients with Lower Urinary Tract Dysfunction: A Single Center Experience from a Developing Country
}

Rehan Mohsin ${ }^{1}$, Altaf Hashmi ${ }^{1}$, Muhammed Mubarak ${ }^{2 *}$, Asad Shehzad ${ }^{1}$, Gohar Sultan ${ }^{1}$, Nazish Ghazanfar ${ }^{1}$, Syed Ali Anwer Naqvi ${ }^{1}$ and Syed Adeeb ul Hassan Rizvi ${ }^{1}$

${ }^{1}$ Urology Department, SIUT, Pakistan

${ }^{2}$ Pathology Department, SIUT, Pakistan

\begin{abstract}
Objectives: Historically, patients with lower urinary tract dysfunction (LUTD) were considered poor candidates for renal transplantation (RT). We aimed to review our experience with this procedure for its safety and efficacy.

Methods: We reviewed the case records of patients with LUTD who underwent RT at our center. Graft and patient survival were analyzed.

Results: Out of 2053 RTs, 26 (1.2\%) patients had LUTD as the primary cause of end-stage renal disease (ESRD). All patients underwent cystourethroscopy prior to transplantation, had abnormal bladders and all underwent bladder augmentation. Only $16(61.5 \%)$ patients had urodynamic (UDN) evaluation prior to transplantation. Pretransplantation augmentation cystoplasty (AC) was performed in $24(92.3 \%)$ patients, and post-RT in two (7.7\%). Mitrofanoff channel was made in $25(96.1 \%)$ patients using appendix in $14(56 \%)$ patients and native ureter in $11(44 \%)$. Double-J (DJ) stents were placed in all patients peroperatively. All patients developed 156 episodes of urinary tract infections (UTIs), with an average of 6 UTIs/ patient. All patients except three are maintaining their graft function within acceptable limits. We observed $100 \%$ patient and graft survival rates in this series.
\end{abstract}

Conclusions: In conclusion, RT combined with AC is a feasible option for patients with LUTD with good results in the medium term and should be explored in selected patients.

Keywords: Augmentation cystoplasty; Graft outcome; Lower urinary tract dysfunction; Renal transplantation; Urodynamics

Abbreviations: BOO: Bladder Outflow Obstruction; CISC: Clean Intermittent Self Catheterization; DJ: Double J; ESRD: End Stage Renal Disease; LUT: Lower Urinary Tract; LUTD: Lower Urinary Tract Dysfunction; PUV: Posterior Urethral Valves; RT: Renal Transplantation; UTI: Urinary Tract Infection; VUR: Vesicoureteral Reflux

\section{Introduction}

Renal transplantation (RT) is considered the treatment of choice for patients with end-stage renal disease (ESRD). There are many causes of ESRD, out of which lower urinary tract dysfunction (LUTD) contributes to approximately $7-20 \%$ of cases in children and adults $[1,2]$. Traditionally, patients with LUTD have been considered poor candidates for RT [3-5]. However, innovative techniques of diagnosis and reconstructive surgery, together with better understanding of the physiological aspects of RTs, excellence in surgical skills, diagnostic tools and the introduction of novel immunosuppressive regimens and antibiotics have led to a better outcome of RTs in LUTD [6-8]. Excellent patient and graft survival rates have been reported in these patient cohorts in different studies. Most of these studies have been reported from centers in the developed world with very few reports from developing countries [5-8]. However, to the best of our knowledge, no such experience with a fair number of patients is available in the literature from Pakistan.

In this study, we analyzed the safety and outcome of RTs in patients with LUTD who underwent bladder augmentation surgery prior to or after RT.

\section{Materials and Methods}

From 1985 to 2011, a total of 3448 RTs were performed at our center. Case records of these patients were analyzed retrospectively for identifying RTs in LUTD with bladder augmentation procedures. A total of 26 such cases were identified who were transplanted for LUTD. First transplant for this indication was performed in 2005. Their case files were scrutinized in detail. The specific causes of renal failure for the patients who had LUTD were noted. Pretransplant urodynamic (UDN) findings and any surgical procedures done were recorded. Peroperative and post-transplant complications were also recorded. Written informed consent was obtained from patients or parents prior to performance of surgical procedures. Standard techniques were used for the RT and the augmentation cystoplasty (AC) in all patients. They all were transplanted kidneys from living-related donors. Standard triple immunosuppressive therapy was used in standard dosages, as described in our previous study [9]. Briefly, the immunosuppressive regimen used at our center comprised of a combination of calcineurin inhibitors (CNIs), anti-proliferative agents and steroids. CNIs included cyclosporine in a dose of $6 \mathrm{mg} / \mathrm{kg} /$ day tapered to $3 \mathrm{mg} / \mathrm{kg} /$ day by the end of 6 months. Tacrolimus was used in high immunological risk groups. Anti-proliferative agents used included azathioprine in a dose of $1.5-2 \mathrm{mg} / \mathrm{kg} / \mathrm{day}$ for standard risk patients and mycophenolate mofetil (MMF) in high risk patients. Steroids were used in a dose of $0.5 \mathrm{mg} / \mathrm{kg} /$ day tapered to $7.5-10 \mathrm{mg} /$ day by the end of three months. Prior to 1991, azathioprine was used in combination with steroids. One patient with 0 haplotype 1 antigen match was induced with

*Corresponding author: Dr. Muhammed Mubarak, Histopathology Department, Sindh Institute of Urology and Transplantation, Karachi-74200, Pakistan, Tel. 009221 9215752; Fax: 009221 2726165; E-mail: info@siut.org

Received September 27, 2012; Accepted September 28, 2012; Published October 10, 2012

Citation: Mohsin R, Hashmi A, Mubarak M, Shehzad A, Sultan G, et al. (2012) Renal Transplantation in Patients with Lower Urinary Tract Dysfunction: A Single Center Experience from a Developing Country. J Transplant Technol Res 2:115. doi:10.4172/2161-0991.1000115

Copyright: $\odot 2012$ Mohsin R, et al. This is an open-access article distributed under the terms of the Creative Commons Attribution License, which permits unrestricted use, distribution, and reproduction in any medium, provided the original author and source are credited. 
antithymocyte globulin (ATG) and six children less than 12 years were induced with interleukin-2 receptor blocker, basiliximab. Renal graft biopsies were performed and interpreted according to Banff 97 classification as described earlier [9]. The patterns of serum creatinine at defined time intervals were analyzed. Episodes of urinary tract infection (UTI) with causative organisms were analyzed. UTI was defined as midstream urine with $\geq 10^{5}$ organisms $/ \mathrm{ml}$ of a single organism irrespective of symptoms [10,11]. Any rejection episodes and the treatment with antithymocyte globulin (ATG) were also recorded. Peroperative placement of double-J (DJ) stent and clean intermittent self-catheterization (CISC) were also recorded. Graft and patient survival were analyzed at last follow-up. A matched control population of 28 non-LUTD renal transplant recipients were analyzed was graft survival. Graft failure was defined as return to dialysis.

\section{Statistical Methods}

Statistical analysis was performed by SPSS version 10.0 (SPSS Inc., Chicago, IL, USA). Data were presented as median \pm interquartile range (IQR) for continuous variables and numbers (percentages) for categorical variables.

\section{Results}

Out of 3448 RTs, 2053 were performed during the last seven years of study (2005-2011). Among the later, 26 (1.2\%) patients underwent RT with LUTD. All of them had undergone bladder augmentation procedures. The demographic characteristics of these patients and specific causes of renal failure (Table 1). The median age of recipients was 21 (IQR: 15-27) years and of donors, 35 (IQR: 25-48) years. All patients were transplanted kidneys from live related donors. All the patients included in this study underwent cystourethroscopy prior to transplantation, and all showed abnormal bladders (thick walled, trabeculations, small capacity, bladder outflow obstruction (BOO)). Only $16(61.5 \%)$ patients had UDN studies prior to RTs, the findings of which are shown in Table 1. AC with ileal patch was carried out before RTs in $24(92.3 \%)$ patients. In two (7.7\%) patients, the procedure was performed after transplantation. Mitrofanoff channel was made in $25(96.1 \%)$ patients using appendix in $14(56 \%)$ patients while native ureter was used in $11(44 \%)$ patients. The duration between RTs and cystoplasty is shown in Table 1 . DJ stents were placed in 22 patient's preoperatively. Postoperative complications of urinary fistula, ureteric obstruction, anastomotic leakage and lymphocoele, one each, were observed in 4 (15.3\%) patients.

The pattern of serum creatinine over time for two years and at the last follow-up and the best serum creatinine values are shown in table 2 . The median lowest serum creatinine was $0.9(0.8-0.99) \mathrm{mg} / \mathrm{dl}$ and was achieved over a median of $6(5-8)$ days. The median serum creatinine at last follow-up was $1.48(1.21-1.92) \mathrm{mg} / \mathrm{dl}$. Regarding infectious complications, all patients developed UTIs with a total number of episodes of 156, and an average of 6 UTIs/patient. Asymptomatic UTIs were seen in $13(50 \%)$ patients with total episodes of 28 UTIs, while symptomatic UTIs were seen in $23(88.46 \%)$ patients with total episodes of 128 UTIs (Table 3 ). The specific microorganisms causing UTIs are shown in Table 3. Renal allograft biopsies were performed in seven patients for unexplained rise in serum creatinine. Two patients showed acute vascular rejection of IIA and IIB types according to Banff classification, two showed acute cellular rejection (type IA), while three patients showed borderline rejection. Only 1 (3.8\%) patient had received ATG for the treatment of II B type of rejection and had UTI. Post-transplant bladder emptying with CISC was performed by 13 patients $(50 \%)$. All these patients also developed both symptomatic and asymptomatic UTIs during CISC. The median follow-up period was 3.09 (IQR: 2.04-3.50 years; range: 0.42-10.23 years) years. Three patients had serum creatinine of $6.31,6.51$ and $6.39 \mathrm{mg} / \mathrm{dl}$ at last follow-up but they are still dialysis free. We thus observed $100 \%$ patient and graft survival in this series till last follow-up. A well matched control population of 28 non-LUTD renal transplant recipients was also analyzed for graft survival during the above study period, which showed $96 \%$ and $72 \%$ graft survival at one and five years respectively.

\section{Discussion}

Patients with congenital or acquired lower urinary tract (LUT)

\begin{tabular}{|c|c|c|}
\hline Demographics of patients & $\mathbf{n}$ & $\%$ \\
\hline Male & 24 & 92.3 \\
\hline Female & 2 & 7.7 \\
\hline M:F ratio & $1: 12$ & \\
\hline Median age (interquartile range) in years & $21(15-27$ & \\
\hline$\leq 18$ years & 11 & 42.3 \\
\hline$>18$ years & 15 & 57.6 \\
\hline \multicolumn{3}{|l|}{ Causes of renal failure } \\
\hline Neurogenic bladder & 11 & 42.6 \\
\hline Posterior uretheral valves & 3 & 11.5 \\
\hline Vesico-ureteric reflux & 6 & 3 \\
\hline Bladder outflow obstruction & 4 & 15.3 \\
\hline Genito-urinary tuberculosis & 2 & 7.6 \\
\hline \multicolumn{3}{|c|}{ Pretransplantation urodynamic findings $(n=16)$} \\
\hline Low capacity low pressure & 5 & 31.2 \\
\hline Low capacity high pressure & 9 & 56.2 \\
\hline Small capacity normal pressure & 2 & 12.4 \\
\hline \multicolumn{3}{|c|}{ Posttransplantation urodynamic findings $(n=20)$} \\
\hline Normal capacity low pressure & 4 & 16.6 \\
\hline Normal capacity normal pressure & 14 & 70 \\
\hline Normal capacity high pressure & 2 & 10 \\
\hline \multicolumn{3}{|c|}{$\begin{array}{l}\text { Duration between cystoplasty and transplantation } \\
\text { in pretransplant cystoplasty }(n=24) \text { in months }\end{array}$} \\
\hline $1-3$ & 4 & 16.6 \\
\hline 4-6 & 5 & 20.8 \\
\hline $7-12$ & 10 & 41.6 \\
\hline $13-18$ & 3 & 12.3 \\
\hline $19-24$ & 2 & 8.3 \\
\hline \multicolumn{3}{|l|}{ Bowel segment used $(n=26)$} \\
\hline lleum & 26 & 100 \\
\hline \multicolumn{3}{|l|}{ Mitrofanoff channel $(n=25)$} \\
\hline Appendix & 14 & 56 \\
\hline Ureter & 11 & 44 \\
\hline
\end{tabular}

Table 1: Demographic and clinicopathological findings in 26 patients with abnormal lower urinary tracts who were transplanted kidneys at our centre.

\begin{tabular}{|l|l|l|}
\hline Serum creatinine values in $\mathbf{~ m g / d l ~}$ & $\begin{array}{l}\text { Median } \\
(\mathbf{I Q R})\end{array}$ & Range \\
\hline Best serum creatinine & $0.9(0.8-0.99)$ & $0.28-2.44$ \\
\hline Normalization of serum creatinine in days & $6(5-8)$ & $4-23$ \\
\hline Serum creatinine at 4 weeks & $1.0(0.89-1.28)$ & $0.35-3.86$ \\
\hline at 3 months & $1.19(0.91-1.49)$ & $0.39-4.36$ \\
\hline at 6 months & $\begin{array}{l}1.1(0.94- \\
1.56)\end{array}$ & $0.45-6.57$ \\
\hline at 12 months & $1.2(1.11-1.53)$ & $0.50-3.10$ \\
\hline at 24 months & $1.6(1.07-1.9)$ & $0.56-2.5$ \\
\hline at last follow-up & $1.48(1.21-1.92)$ & $0.70-6.51$ \\
\hline Duration of follow-up in years & $3.09(2.04-3.5)$ & $0.42-10.23$ \\
\hline
\end{tabular}

Table 2: Pattern of serum creatinine over time in 26 patients with LUTD who underwent renal transplantation. 


\begin{tabular}{|l|l|l|}
\hline Total episodes of UTIs & 156 & \\
\hline Mean UTIs/patient & 6 & \\
\hline Median (interquartile range) & $5(3-8)$ & \\
\hline Range of UTIs/patient & $1-19$ & \\
\hline Micro-organisms causing UTI & $\mathrm{N}$ & $\%$ \\
\hline E.Coli & 74 & 47.3 \\
\hline Klebsiella & 35 & 22.4 \\
\hline Pseudomonas & 17 & 10.89 \\
\hline Morgnella & 10 & 6.4 \\
\hline Acinetobacter & 8 & 5.1 \\
\hline Enterococcus & 8 & 5.1 \\
\hline Others & 7 & 4.4 \\
\hline
\end{tabular}

Table 3: Urinary tract infection (UTI) episodes in all 26 patients and the causative organisms.

disease frequently develop ESRD requiring renal replacement therapy. Abnormal LUTs of these patients should be managed surgically or sometimes conservatively to preserve normal renal functions. However, patients developing ESRD are scheduled for RTs and augmented procedures to make the reservoir more optimal to sustain the precious renal graft function $[3,4]$.

We herein report our experience with 26 patients who underwent RT for ESRD secondary to LUTD, out of which 24 (92.3\%) patients had AC before RTs. The advantages of pretransplantation AC include avoidance of the interaction of the augmentation surgery with immunosuppressive drugs and the facilitation of antireflux mechanisms [5]. Contrary to this, Mc Inerny et al. advocate the policy to perform cystoplasty after RT to avoid dry reservoir, interference with its vascular pedicle at the time of ureteric implantation and to allow renal functions to stabilize [6].

The timing of cystoplasty before RT varies in the literature from $10-12$ weeks to 5 years $[5,7]$. We strongly advocate pretransplantation $\mathrm{AC}$ at our center. In this series, we performed $24 \mathrm{AC}$ before RT and the time period between AC and RT was $>6$ months in the majority of cases $(62.5 \%)$ (Table 1$)$. The main reason for this somewhat extended time interval was lack of interest for organ donation on the part of the family. In this part of the world there is no deceased donor programme and there are multiple social issues which hinder kidney donation among families.

To achieve the most optimal results in RT especially in LUTD patients, pretransplant LUT UDN studies should be performed with cystoscopy so that any correctable or palliative surgical procedure could be offered to these patients. We performed UDN in 16 patients before RT. The majority of these patients (56.2\%) had low capacity and high pressure urinary bladders and 4 patients had low capacity and low pressure bladders (Table 1). All these patients were managed with preRT AC. Later, the majority of these patients had LUT UDN in post-RT phase, which showed an adequate volume and pressure in the LUT, which was in concordance with an earlier study [8]. This low pressure and adequate volume of LUT provides an environment for the allograft to function in an optimal manner.

UTI in RT patients is a major concern for the graft well being and can increase the morbidity. The overall incidence of UTI in RT patients varies from $30 \%$ to $65 \%$ [10-14]. Multiple factors affect UTI in RT: among these, DJ stent placement is a major factor and provides an excellent nidus for the colonization of the foreign body in the immunosuppressed patients. The incidence of UTIs with DJ stenting is markedly high and varies between 14 to $71 \%[11,15,16]$. Augmented bladders also provide a favorable atmosphere for the occurrence of UTIs. The incidence of UTIs in RTs with augmented bladders has been reported to be up to $100 \%$ [17]. We also observed an incidence of UTIs of $100 \%$ with augmented bladders and DJ stenting, which is comparable to that observed in the above mentioned studies.

In this cohort, $50 \%$ of the patients required CISC and managed it successfully. All of these patients had at least one episode of UTI with significant episodes of symptomatic UTIs. UTI in CISC is not an infrequent finding owing to the bladder enlargement and repeated introduction of catheters in Mitrofanoff channel. Personal hygiene and social factors also play a part in the occurrence of UTIs in CISC. The combination of CISC, augmented bladders and DJ stenting are the strong risk factors for UTIs but the important point to be emphasized here is to construct a technically correct antireflux ureteric reimplant to prevent graft pyelonephritis [8] and to maintain graft function in normal and acceptable range.

Gill et al. reported UTIs with CISC in RT of 33\% [18], which is somewhat low as compared to other studies, which showed higher incidence [19]. We report $100 \%$ incidence of UTIs, but we think that the objective of CISC in Mitrofanoff channel should be the complete and frequent emptying of the bladder under sterilized measures. This coupled with a good antireflux ureteric reimplant technique may minimize the chances of UTIs and graft pyelonephritis which is a requirement for the better graft survival. We therefore advocate CISC in selected cases despite high incidence of UTI as frequent CISC is a requirement to keep a high LUT pressure under control to minimize the chances of graft hydronephrosis and maintain an optimal graft function.

A total of 156 episodes of UTI were documented among these patients in this study. The maximum numbers of UTIs in a single patient was 19. E. coli was the most common organism isolated in this study followed by Klebsiella, Pseudomonas and Morganella. Multiple studies have shown high incidence of UTI with E. coli and Klebsiella [11,20-22]. This high incidence of UTIs led the transplant physicians to stringent follow-up of these patients and diagnostic protocols in which urine analysis and serum creatinine monitoring are mandatory.

The median best serum creatinine in this series was less than 1.0 $\mathrm{mg} / \mathrm{dl}$ with an excellent graft and patient survival as compared with a small control population of 28 patients well matched for all other parameters except LUTD. This excellent graft survival has also been substantiated by other authors and may be due to more rigorous follow-up and prompt treatment [8, 22-26]. However, this study has some limitations as graft function depends on multiple factors and these factors should be assessed carefully. The follow-up period is also not very long. The study originates from a single center. Despite these limitations, we believe that our results are promising and support the safety and efficacy of RT in this group of patients.

\section{Conclusions}

In conclusion, our results show that $\mathrm{AC}$ is a safe and feasible procedure in patients with LUTD and RTs can be done in these patients under a multidisciplinary care with excellent patient and graft survival in the medium term.

\section{References}

1. Ali-El-Dein B, Abol-Enein $\mathrm{H}$, El-Husseini A, Osman $\mathrm{Y}$, Shehab El-Din AB, et al. (2004) Renal transplantation in children with abnormal lower urinary tract. Transplant Proc 36: 2968-2973.

2. Koo HP, Bunchman TE, Flynn JT, Punch JD, Schwartz AC, et al. (1999) Renal Transplantation in children with severe lower urinary tract dysfunction. J Uro $161: 240-245$. 
Citation: Mohsin R, Hashmi A, Mubarak M, Shehzad A, Sultan G, et al. (2012) Renal Transplantation in Patients with Lower Urinary Tract Dysfunction: A Single Center Experience from a Developing Country. J Transplant Technol Res 2:115. doi:10.4172/2161-0991.1000115

3. Kelly WD, Merkel FK, Markland C (1966) lleal urinary diversion in conjunction with renal homotransplantation. Lancet 1:222-226.

4. Marshall FF, Smolev JK, Spees EK, Jeffs RD, Burdick JF (1982) The urological evaluation and management of patients with congenital lower urinary tract anomalies prior to renal transplantation. J Urol 127: 1078-1081.

5. Riedmiller H, Gerharz EW, Köhl U, Weingärtner K (2000) Continent urinary diversion in preparation for renal transplantation: a staged approach. Transplantation 70: 1713-1717.

6. Mclnerney PD, Picramenos D, Koffman CG, Mundy AR (1995) Is cystoplasty a safe alternative to urinary diversion in patients requiring renal transplantation? Eur Urol 27: 117-120.

7. Marechal JM, Sanseverino R, Gelet A, Martin X, Salas M, et al. (1990)Continent cutaneous ileostomy (Kock pouch) prior to renal transplantation. Br J Urol 65 317-321.

8. Nahas WC, Lucon M, Mazzucchi E, Antonopoulos IM, Piovesan AC, et al. (2004) Clinical and urodynamic evaluation after ureterocystoplasty and kidney transplantation. J Urol 171: 1428-1431.

9. Kazi JI, Mubarak M (2012) Biopsy findings in renal allograft dysfunction in a live related renal transplant program. J Transplant Tech Res 2:108.

10. Elkehili IM, Kekli AB, Zaak AS, Salem EL (2010) Urinary tract infection in rena transplant recipients. Arab J Nephrol Transplant 3:53-55.

11. Iqbal T, Naqvi R, Akhter SF (2010) Frequency of urinary tract infection in renal transplant recipients and effect on graft function. J Pak Med Assoc 60: 826-829.

12. Rivera-Sanchez R, Delgado-Ochoa D, Flores-Paz RR, García-Jiménez EE, Espinosa-Hernández R, et al. (2010) Prospective study of urinary tract infection surveillance after kidney transplantation. BMC Infect Dis 10: 245.

13. Golebiewska J, Debska SA, Komarnicka J, Samet A, Rutkowski B (2011) Urinary tract infections in renal transplant recipients. Transplant Proc 43:29852990.

14. Rice JC, Peng T, Kuo YF, Pendyala S, Simmons L, et al. (2006) Renal allograft injury is associated with urinary tract infection caused by Escherichia coli bearing adherence factors. Am J Transplant 6: 2375-2783.
15. Fayek SA, Keenan J, Haririan A, Cooper M, Barth RN, et al. (2012) Uretera stents are associated with reduced risk of ureteral complications after kidney transplantation: a large single center experience. Transplantation 93:304-308.

16. Ranganathan M, Akber M, Ilham MA, Chavez R, Kumar N, et al. (2009) Infective complications associated with ureteral stents in renal transplant recipients. Transplant Proc 41: 162-164.

17. Flood HD, Malhotra SJ, O'Connell HE, Ritchey MJ, Bloom DA, et al. (1995) Long-term results and complications using augmentation cystoplasty in reconstructive urology. Neurourol Urodyn 14:297-309.

18. Gill IS, Hayes JM, Hodge EE, Novick AC (1992) Clean intermitten catheterization and urinary diversion in the management of renal transplant recipients with lower urinary tract dysfunction. J Urol148: 1397-1400.

19. Akoh JA, Choon TC, Akyol MA, Kyle K, Briggs JD (1999) Outcome of renal transplantation in patients with lower urinary tract abnormality. J R Coll Surg Edinb 44: 78-81.

20. Mitra S, Alangaden GJ (2011) Recurrent urinary tract infections in kidney transplant recipients. Curr Infect Dis Rep 13:579-1587.

21. Moradi M, Abbasi M, Moradi A, Boskabadi A, Jalali A (2005) Effect of antibiotic therapy on asymptomatic bacteriuria in kidney transplant recipients. Urol $\mathrm{J} 2$ : $32-35$

22. Pereira DA, Barroso U Jr, Machado P, Pestana JO, Rosito TE, et al. (2008) Effects of urinary tract infection in patients with bladder augmentation and kidney transplantation. J Urol 180: 2607-2610

23. Blanco M, Medina J, Pamplona M, Miranda N, Gonzalez E, et al. (2009) Outcome of renal transplantation in adult patients with augmented bladders. Transplant Proc 41: 2382-2384.

24. Bilginer Y, Aki FT, Topaloglu R, Tekgul S, Demirkaya E, et al. (2008) Renal transplantation in children with lower urinary tract dysfunction of different origin: a single-center experience. Transplant Proc 40: 85-86.

25. Otukesh H, Sharifian M, Simfroosh N, Basiri A, Hoseini R, et al. (2005) Outcome of renal transplantation in children with lower urinary tract abnormality. Transplant Proc 37: 3071-3074.

26. Taghizadeh AK, Desai D, Ledermann SE, Shroff R, Marks SD, et al. (2007) Renal transplantation or bladder augmentation first? A comparison of complications and outcomes in children. BJU Int 100: 1365-1370. 